\title{
Laparoscopic complications of gynecologic procedures
}

\begin{abstract}
Complications of gynecologic laparoscopic procedures are relatively uncommon but may be devastating. These complications occur approximately in 5 per 1000 procedures and majority of them are encountered during the entry stage of the surgery. Vascular structures, gastro-intestinal organs and urinary tract are the most common affected sites. There are several patients and surgeon related factors that might influence complications, however in the presence of adequate expertise, most of these complications can be avoided. Not only prevention, but also early detection is of importance during laparoscopic procedures.
\end{abstract}

Keywords: laparoscopy, gynecology, complication
Volume 4 Issue I - 2016

\section{Emre Goksan Pabuccu}

Department of Obstetrics and Gynecology, Ufuk University School of Medicine, Turkey

Correspondence: Emre Goksan Pabuccu, Department of Obstetrics and Gynecology, Ufuk University School of Medicine, Mevlana Bulvar No 86, Ankara, Turkey, Tel 90-312-204-4000, +90-532-4I4-7844, Email pabuccu@hotmail.com

Received: August 19,2015 | Published: January 09, 2016

\section{Introduction}

As the number of endoscopic procedures has increased tremendously over the years, several different complications may arise affecting different systems. More than approximately half of these complications occur during entry; while the rest occurs during or after the operation. The majority of studies related to this subject revealed that the rate of complications decrease by increasing surgical experience. ${ }^{1,2}$ Complications are generally classified as; entry stage, intra-operative or post-operative complications.

\section{Complications during the entry stage}

More than half of laparoscopic complications obviously occur during the entry stage. As the entry is a relatively blind procedure, it also brings severe risks along. Complications with respect to the entry technique occur in approximately $0.3-1 \%$ of the entire laparoscopic procedures and the rate of mortality is approximately 7 in $1,00,000{ }^{3,4}$ At this stage, especially vascular, urinary and intestinal-gastric injuries can be observed. Primarily, 3 entry techniques are used for diagnostic or operative laparoscopic procedures; entry following $\mathrm{CO} 2$ insufflation with Verres needle (closed), direct trocar entry and open Hasson's technique. All techniques have their own advantages and also several different risks for each of these entries are obviously available. At this stage, proper elevation of the abdominal wall is of importance to preclude complications. In order to elevate the abdominal wall, manual elevation (Figure 1A), elevation from umbilical edges with clamps (Figure 1B) and elevation from $2 \mathrm{~cm}$ lateral to the umbilicus with clamps (Figure 1C) are frequently used. It was reported that the method clearing the distance between the abdominal wall and viscera among these 3 methods is the method of elevation from umbilical edges with clamps. ${ }^{5}$

When this method is used, the distance between the needle and viscera is approximately $6.8 \mathrm{~cm}$ and the force applied while inserting trocar does not change this distance. However, the distance becomes less by manual elevation or laterally $2 \mathrm{~cm}$ elevation with clamps. ${ }^{6}$

There are several methods available to predict whether the Verres needle is properly inserted into the abdominal cavity or not (Figure $2 \mathrm{~A} \& 2 \mathrm{~B})$ :

Previous studies reported that the abdomen was penetrated by a success of $85 \%$ on the first trial of Verres needle insertion and 2 or more trials are needed for approximately $8 \%$ of the cases. ${ }^{7}$ The rate of complication was $0.8 \%$ on the first trial; this rate was determined as $44 \%$ on the third trial and as $84.6 \%$ on more trials. If the abdomen is unable to be successfully penetrated on 3 trials by Verres needle, alternative entries must be considered because of the prediction that a prominent increase will be among complication risks.

In cases with the doubt of technical difficulty, entry can be carried out on the mid-clavicular line called Palmer's point, approximately $3 \mathrm{~cm}$ inferior to the left costa; possible several injuries may be prevented by using this localization. Palmer's point can be used for penetrating with Verres needle in cases with especially the presence of adhesion, previous hernia repair, obesity, abdominal wall relaxation, the presence of a large mass and pregnancy. Despite the measures counted, various injuries may be observed during entry. Intestinal and retroperitoneal vascular injuries constitute the majority of complications observed during entry. Following the entry procedure, injury regions according to the percentage of frequency can be counted as: small intestine (25\%), iliac artery $(19 \%)$, colon $(12 \%)$, iliac or other retroperitoneal veins (9\%), mesenteric artery (7\%), aorta $(6 \%)$, vena cava inferior $(4 \%)$, abdominal wall arteries $(4 \%)$, bladder $(3 \%)$ and liver $(2 \%){ }^{8}$

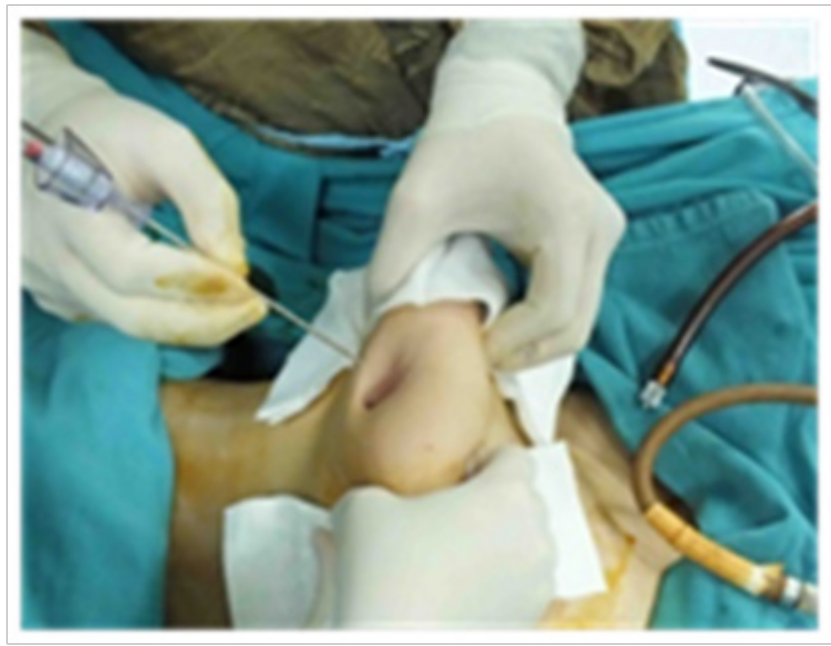

Figure IA Manual elevation of abdominal wall. 


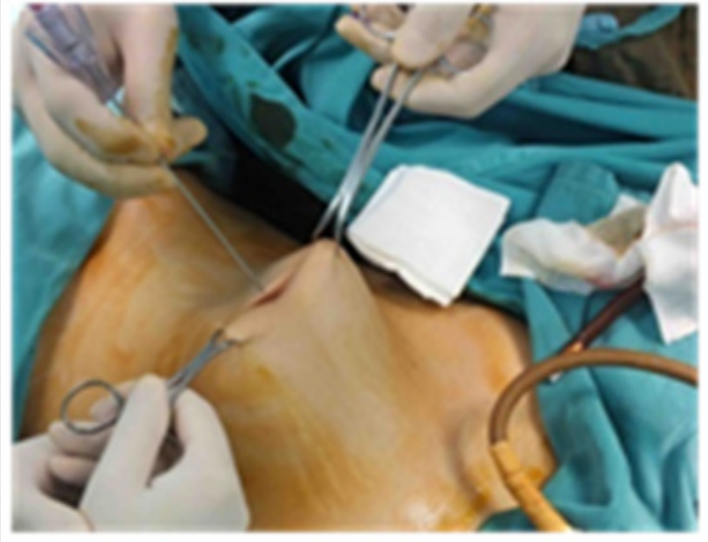

Figure IB Elevation from umbilical edges with clamps.

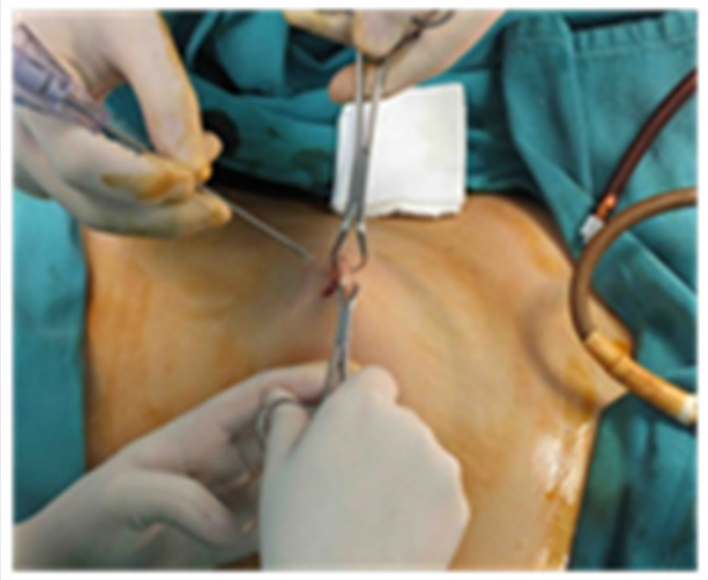

Figure IC Elevation from $2 \mathrm{~cm}$ lateral to the umbilicus with clamps.

i. Double 'click' sound while penetrating.

ii.Aspiration test with injector after penetrating with Verres needle.

iii. Negative pressure test applied by I drop of isotonic into the posterior lumen of the Verres needle after entry (Figure 2A).

iv. Insufflation pressure $<8 \mathrm{mmHg}$ and easy gas flow.

v. Following gas flow, receiving tympanic sound by infra-hepatic percussion (Figure 2B).

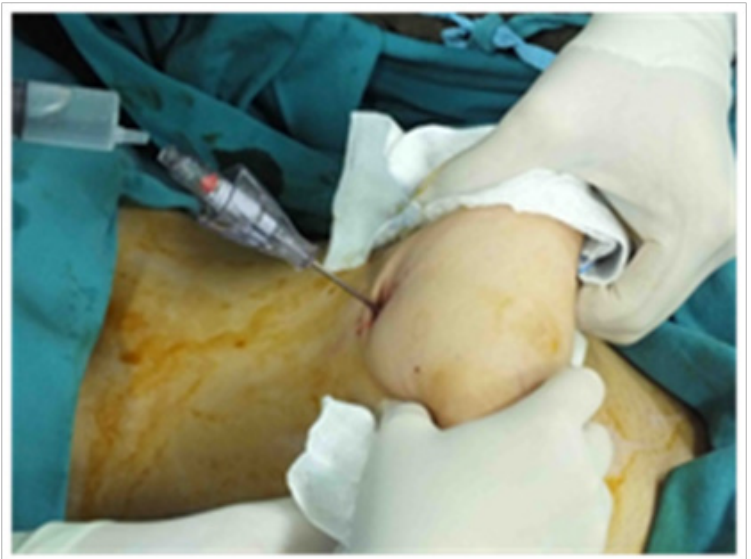

Figure 2A Negative pressure test applied by I drop of isotonic into the posterior lumen of the Verres needle after entry.

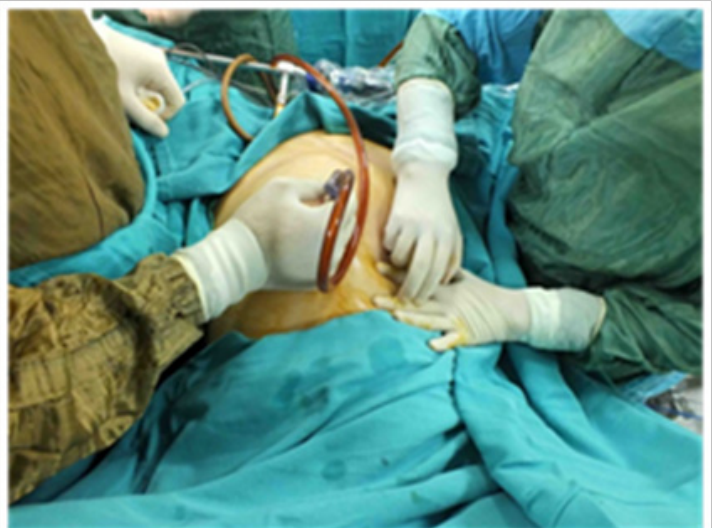

Figure 2B Gas flow, receiving tympanic sound by infra-hepatic percussion.

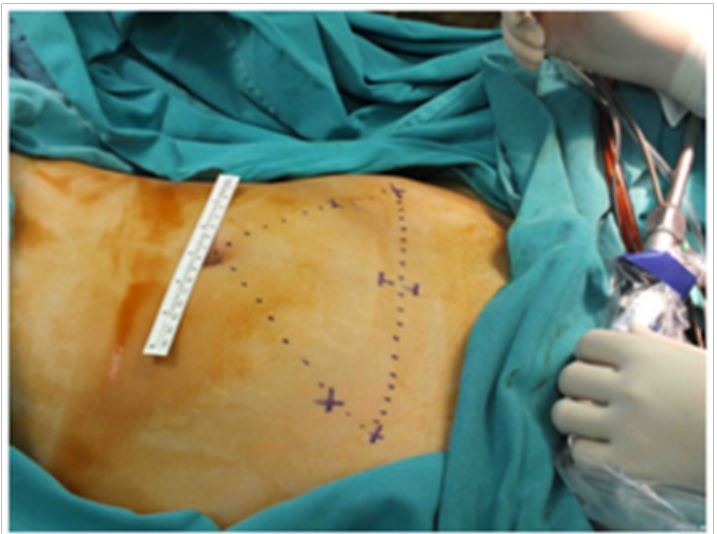

Figure 3 The most appropriate region in many cases is $1.25 \mathrm{~cm}$ superior or inferior to the imaginary line from the iliac eminentia.

As well as being the rarest, major vascular complications occurring after closed or direct trocar entry are observed in approximately $0.04-0.1 \%$ of laparoscopic procedures. ${ }^{9,10}$ Despite low prevalance, the rate of mortality is $9-17 \%$ in the presence of major vascular complications. ${ }^{3}$ These injuries, which can generally occur during the entry by Verres needle or large trocar are observed in the 2nd or 3rd trial following unsuccessful intervention. ${ }^{8}$ Abundant bleeding behind the needle after Verres needle entry is probably due to major vascular trauma. In this case, performing emergency laparotomy without dislocating the needle, efficient buffering and requesting immediate cardiovascular surgery consultation is crucial.

During laparoscopic entry, gastrointestinal system injuries may also be observed $(0.06 \%) .{ }^{11}$. The risk increases even more, especially with a peritonitis history or the presence of an adhesion. Cruise of filling pressure above $8-10 \mathrm{mmHg}$, asymmetric distention of the peritoneal cavity, observing gastric or intestinal content from Verres lumen are likely associated with intestinal injury. Small Verres injuries may sometimes be managed by follow-up only; however, if the lesion is large or occurs following direct trocar entry, a surgical method may be required. Large gastrointestinal injuries may result in diffuse intraabdominal infection and sepsis, as delayed diagnosis contributes to morbidity and mortality prominently. Half of the entry-related intestinal injuries show signs at least after 24 hours. ${ }^{11}$ Generally, laparotomic approach is adopted in the management of these cases $(63 \%)$; mortality may occur in approximately 5\% despite efficient 
follow-up and therapy. ${ }^{3,4}$ According to the degree of injury, primary repair, segmental resection-anastomosis or colostomy procedures may be required.

Table I Comparison of benefits

\begin{tabular}{|c|c|c|c|c|}
\hline & $\begin{array}{l}\text { Sensitivity } \\
\%\end{array}$ & $\begin{array}{l}\text { Specificity } \\
\%\end{array}$ & $\begin{array}{l}\text { Positive } \\
\text { predictive } \\
\text { value \% }\end{array}$ & $\begin{array}{l}\text { negative } \\
\text { predictive } \\
\text { value } \%\end{array}$ \\
\hline $\begin{array}{l}\text { Double Click } \\
\text { Test }\end{array}$ & 39 & 69 & 19 & 86 \\
\hline Aspiration Test & 0 & 98 & 0 & 83 \\
\hline Drop Test & 16 & 89 & 22 & 85 \\
\hline $\begin{array}{l}\text { Intraabdominal } \\
\text { Pressure }<10 \\
\mathrm{mmHg}\end{array}$ & 79 & 63 & 32 & 93 \\
\hline
\end{tabular}

\section{Complications during operation}

Several complications may also be encountered following laparoscopic penetration. These may be as follows: vascular, gastrointestinal, urinary injuries or injuries of some systems based on the usage of thermal energy. Vascular injuries are generally minor which are likely to be observed during trocar insertions and are generally avoided by using the transluminance technique. However, large vascular injuries may also be encountered during large or lateral trocar penetrations.

Major Vascular Injuries: Despite low prevalence (0.04-0.5\%), approximately $20 \%$ of the cases may result in mortality. ${ }^{12}$ The most common injury sites during an operation are aorta, vena cava and iliac arteries. There are several risk factors contributing to the complications. In the report of Baggish et al. ${ }^{13}$ majority of the cases $(71 \%)$ where major vascular complications were encountered was overweight or obese patients. ${ }^{13}$ Furthermore, inadequate surgical experience and inappropriate patient positioning may also contribute to increased complication rates. It is crucial to determine the site of the aortic bifurcation conjecturally before starting the procedure. This region may not always be palpated or detected according to the umbilicus. The most appropriate region in many cases is $1.25 \mathrm{~cm}$ superior or inferior to the imaginary line from the iliac eminentia (Figure 3).

Major Intra-operative vascular injuries are frequently observed in more complicated procedures and in cases with adhesion formation. Such procedures as lymph node and retroperitoneum dissections or sacral colpo-pexia procedure are relatively risky due to anatomic localizations. Sudden and major bleeding following penetration with Verres needle/trocar or intra-abdominal growing hematoma especially in the retroperitoneal region following thermal energy usage is most likely to be due to a major injury. According to the injury site and dimension, laparoscopic repair may be preferred mostly depending on surgical experience. If there is a suspicion of injury during penetration, reaching the injury site through midline laparotomy and planning repair without removing trocar is a more appropriate approach; because it will be more difficult to reach the repair area by abundant bleeding when trocar is removed (Table 1).

Intestinal injuries: The incidence of intestinal injuries is approximately $0.5 \%$ and near one half of these complications occur intra-operatively. ${ }^{14}$ Small intestine and colon (recto-sigmoid) are the most frequent regions of injury mostly due to inappropriate use of the thermal energy. Because the injuries during the operation are generally not recognized, a delay in diagnoses generally contributes to peritonitis, severe intra-abdominal infections and sepsis. Typically, the most frequent reason of laparoscopy-related mortalities is accepted as intestinal injuries due to delayed diagnosis..$^{15,16}$ The most predictive factors in these complications are: technical difficulty of the surgical procedure, condition of the case and surgeon's experience. In the review of Brosens \& Gordon. ${ }^{17}$ it was reported that the reliability of surgeon increases where complications decrease as a consequence of performing 100 laparoscopic procedures yearly. ${ }^{17}$

Aspiration of the intestinal content from Verres needle, smelling faecaloid from trocar areas or notable faecaloid coming from trocar is apparent signs of an injury. Intra-abdominal pressure during penetration to be between 8 and $10 \mathrm{mmHg}$ may also be an indirect indicator. If rectosigmoid injury is suspected during the operation, intestinal integrity may be tested. In this test, after pelvis is filled with isotonic, air is given into rectum by $60 \mathrm{ml}$ catheter type injector. Also the proximal sigmoid colon is compressed laparoscopically and observed whether there is an air bubble on behalf of a possible leakage in the rectum. However, the injury may not always be determined clearly and morbidites may be observed due to delayed diagnosis. Clinical findings are generally nonspecific in the postoperative period and may be presented as mild abdominal pain, subfebrile fever, and nausea. However, the overall diagnosis time of occult injuries range between 1-21 days.

Urinary injuries: The incidence of urinary injuries during laparoscopic procedures is approximately $0.2 \%$ and this rate is very similar with that of observed in laparotomic procedures. Mainly, ureter and bladder injuries may occur. In order to avoid these complications, it is crucial to clarify the pelvic anatomy, especially the trace of ureter in the pelvis. In laparoscopic procedures, the bladder is most likely to get injured and these injuries are recognized more rapidly compared to ureter injuries. ${ }^{18}$ Previous pelvic surgeries, infections, the presence of endometriosis, pelvic tumors and all kinds of factors impairing anatomical integrity may contribute to iatrogenic ureter injuries.

Ureter is one of the common sites of laparoscopic complications. Because of increasing numbers of laparoscopic hysterectomy procedures, ureter injuries are encountered more often when compared to abdominal hysterectomies. Ureter injury risk is determined as 2.6-35 times higher with laparoscopic hysterectomy compared to laparotomy. ${ }^{19}$ The part, which is adjacent to the infindibulo-pelvic ligament in the pelvic inlet of the ureter, is the common injury site especially in laparoscopic-assisted vaginal hysterectomy operations. ${ }^{16}$ Injuries are generally secondary to thermal energy related transsection, ligation, retraction, contusion, devascularization and are frequently not noticed. Patients may apply within the first 3 days due to injury-induced symptoms and sometimes it takes couple of weeks after the operation. The majority of injuries $(70 \%)$ are detected in the postoperative period; however $20 \%$ of these injuries may not be recognized.

Bladder injuries are generally observed during suprapubic trocar penetration, in the bladder dissection or as a result of excessive thermal energy use. However, as the majority of these injuries are detected during the operation $(90 \%)$, morbidity rates are low. In suspicious cases, the bladder can be filled with methylene blue and the presence of leakage could be examined. In the presence of an injury, observation without any intervention or primary repair can be considered. In small injuries, repair with single-layer $2 / 0-3 / 0$ absorbable sutures along with continuous urine drainage (7-10 days) is generally adequate. Especially in larger injuries $(>1 \mathrm{~cm})$ or in thermal energy injuries, 2 layer repair is suggested. ${ }^{19}$ 


\section{Postoperative complications}

Hernia formation in trocar region: When compared to laparatomic surgery, one of the most significant advantages of laparoscopy is to enable decrease in postoperative hernia formation. The incidence is between 0.06 and $1 \%$ and this rate is approximately 10-100 times lower when compared with laparotomy. ${ }^{14}$ This complication is due to improper and inadequate closure of trocar penetration sites and is somehow preventable. Hernia formation generally occurs in trocar inlets that are larger than $5 \mathrm{~cm}$, however they may also be observed in small trocar sites. Small intestine, rarely colon and omentum are found as content in hernias that are observed in extraumbilical regions where a large trocar is used. Especially in undetected hernias, the risk for intestinal incarceration may be as high as $20 \% .{ }^{20}$ In order to avoid hernia formation, using $10 \mathrm{~mm}$ trocar instead of $12 \mathrm{~mm}$, preferring those with blunt-conic tip instead of sharp tip, repairing fascias, removing small trocars in guidance of a camera, and closing the valve mechanism while removing are recommended. Hernia repairs are generally performed laparotomically; however, laparoscopy may also be used.

\section{Conclusion}

As a result of increasing request for minimal invasive procedures, a growing number of laparoscopic procedures are carried out today. The essential principle should be to increase the expertise in order to protect from these complications. This can also be possible by assistant training programs and post-graduate courses. In this context, using and promoting simulators for training can be attributed as a simple but effective step for laparoscopic experience.

\section{Acknowledgments}

None.

\section{Conflicts of interest}

The authors declare there is no conflict of interests.

\section{Funding}

None.

\section{References}

1. Magrina JF. Complications of laparoscopic surgery. Clin Obstet Gynecol. 2002;45(2):469-480.

2. Makai G, Isaacson K. Complications of gynecologic laparoscopy. Clin Obstet Gynecol. 2009;52(3):401-411.

3. Lehmann-Willenbrock E, Riedel HH, Mecke H, et al. Pelviscopy/ laparoscopy and its complications in Germany, 1949-1988. J Reprod Med. 1992;37(8):671-677.
4. Hulka J, Peterson HB, Phillips JM, et al. Operative laparoscopy: American Association of Gynecologic Laparoscopists' 1993 membership survey. J Am Assoc Gynecol Laparosc. 1995;2(2):133-136.

5. Roy GM, Bazzurini L, Solima E, et al. Safe technique for laparoscopic entry into the abdominal cavity. $J$ Am Assoc Gynecol Laparosc. 2001;8(4):519-528.

6. Teoh B, Sen R, Abbott J. An evaluation of four tests used to ascertain Veres needle placement at closed laparoscopy. J Minim Invasive Gynecol. 2005;12(2):153-158.

7. Sogc Clinical Practice Guideline. Laparoscopic Entry Review of Technique, Technologies and Complications. 2007;No:193.

8. Yuzpe AA. Pneumoperitoneum needle and trocar injuries in laparoscopy. A survey on possible contributing factors and prevention. J Reprod Med. 1990;35(5):485-490.

9. Hashizume M, Sugimachi K. Needle and trocar injury during laparoscopic surgery in Japan. Surg Endosc. 1997;11(12):1198-1201.

10. Hasson HM. A modified instrument and method for laparoscopy. Am J Obstet Gynecol. 1971;110(6):886-887.

11. Chandler JG, Corson SL, Way LW. Three spectra of laparoscopic entry access injuries. J Am Coll Surg. 2001;192(4): 478-490.

12. Tarik A, Fehmi C. Complications of gynaecological laparoscopy-a retrospective analysis of 3572 cases from a single institute. J Obstet Gynaecol. 2004;24(7):813-816.

13. Baggish MS. Analysis of 31 cases of major-vessel injury associated with gynecologic laparoscopic operations. J Gynecol Surg. 2003;19(2):63-73.

14. Chapron C, Pierre F, Harchaoui Y, et al. Gastrointestinal injuries during gynaecological laparoscopy. Hum Reprod. 1999;14(2):333-337.

15. Crist DW, Gadacz TR. Complications of laparoscopic surgery. Surg Clin North Am. 1993;73(2):265-289.

16. Peterson HB, Hulka JF, Phillips JM. American Association of Gynecologic Laparoscopists' 1988 membership survey on operative laparoscopy. J Reprod Med. 1990;35(6):587-589.

17. Brosens I, Godron. A Bowel injuries during gynaecological laparoscopy: a multinational survey. Gynaecol Endosc. 2001;10(3):141-145.

18. Hasson HM, Parker WH. Prevention and management of urinary tract injury in laparoscopic surgery. J Am Assoc Gynecol Laparosc. 1998;5(2):99-114.

19. Härkki-Siren P, Sjoberg J, Kurki T. Major complications of laparoscopy: A follow-up Finnish study. Obstet Gynecol. 1999;94(1):94-98.

20. Berker B, Taskın S, Taskın E. Complications of Laparoscopic Gynecologic Surgery. In: Michael S, editors. Prevention and Management of Laparoendoscopic Surgical Complications. 3rd edn. Society of Laparoendoscopic Surgeons, USA; 2012. 